\title{
POSITIVITY OF THE WEIGHTS OF EXTENDED CLENSHAW-CURTIS QUADRATURE RULES
}

\author{
TAKEMITSU HASEGAWA, HIROSI SUGIURA, AND TATSUO TORII
}

\begin{abstract}
We prove that some extended Clenshaw-Curtis quadrature rules have all weights positive. We also present extended Filippi rules of open type having all weights positive. Conjectures on the possibility of other positive quadrature rules embedded in Clenshaw-Curtis or Filippi rule are suggested.
\end{abstract}

\section{INTRODUCTION}

In this paper we shall establish that some integration rules embedded in the Clenshaw-Curtis rule [4] (henceforth abbreviated to CC rule) proposed in [16] have all their weights positive. This is important to guarantee the numerical stability and convergence of the quadrature rules $[9$, p. 189], [14, p. 335]. For additional literature on positive quadrature rules, we refer to $[1,3,5,7,11,12$, $20,21,22]$.

The CC rule is an interpolatory integration rule which approximates the integral $Q(f)=\int_{-1}^{1} f(x) d x$ by

$$
Q_{N}(f)=\int_{-1}^{1} L_{N}(f ; x) d x=\sum_{i=0}^{N} w_{N i} f\left(x_{N i}\right),
$$

where $L_{N}(f ; x)$ denotes the unique polynomial of degree less than or equal to $N$ interpolating a given smooth function $f(x)$ at $N+1$ points $x_{N i}$ :

$$
L_{N}\left(f ; x_{N i}\right)=f\left(x_{N i}\right), \quad 0 \leq i \leq N .
$$

The double prime in (1.1) denotes the summation where the first and last terms are halved.

The abscissae $x_{N i}=\cos (\pi i / N), 0 \leq i \leq N$, are the zeros of an auxiliary polynomial $\omega_{N+1}(x)$ of degree $N+1$ defined by

$$
\omega_{N+1}(x)=T_{N+1}(x)-T_{N-1}(x)=2\left(x^{2}-1\right) U_{N-1}(x) .
$$

In (1.3), $T_{k}(x)$ and $U_{k}(x)$ denote the Chebyshev polynomials of the first and second kinds, respectively, and are given by $T_{k}(x)=\cos k \theta$ and $U_{k}(x)=$ $\sin (k+1) \theta / \sin \theta \quad(x=\cos \theta)$. If the function $f(x)$ is sufficiently smooth, the approximation $Q_{N}(f)$ (1.1) converges rapidly as $N$ increases.

Received by the editor February 27, 1991 and, in revised form, May 19, 1992.

1991 Mathematics Subject Classification. Primary 65D30, 65D32.

Key words and phrases. Extended Clenshaw-Curtis rule, numerical integration, positivity of weights. 
From (1.2) and (1.3) we have the Lagrange form for $L_{N}(f ; x)$,

$$
L_{N}(f ; x)=\sum_{i=0}^{N} l_{N i}(x) f\left(x_{N i}\right),
$$

where $l_{N i}(x)$ is the polynomial

$$
l_{N i}(x)=\frac{e_{i} \omega_{N+1}(x)}{\left(x-x_{N i}\right) \omega_{N+1}^{\prime}\left(x_{N i}\right)}=\frac{2}{N} \sum_{k=0}^{N} T_{k}\left(x_{N i}\right) T_{k}(x), \quad 0 \leq i \leq N,
$$

$e_{i}=1(1 \leq i<N), e_{0}=e_{N}=2$, and $\omega_{N+1}^{\prime}(x)$ denotes the derivative of $\omega_{N+1}(x)$. For the derivation of the second equality above, see $[4,16]$.

Imhof [18] proved that the weights $w_{N i}=\int_{-1}^{1} l_{N i}(x) d x$ in (1.1) are all positive. Furthermore, it is well known that the sequence $\left\{Q_{N}(f)\right\}$ of the quadrature rules can be generated recursively by doubling $N$ and efficiently by using the Fast Fourier Transform (FFT) [2, 13, 24]. In [16] we showed that it is possible to increase $N$ more slowly than doubling, thereby enhancing the economy of an automatic quadrature [6, p. 418] without sacrificing the accuracy. To be specific, the sequence of $N$ is

$$
N=6,8,10, \ldots, 3 \times 2^{n}, 4 \times 2^{n}, 5 \times 2^{n}, \ldots, \quad n=1,2, \ldots,
$$

and the abscissae for $Q_{N}(f)$ are chosen so that the sequence $\left\{Q_{N}(f)\right\}$ is an embedded sequence [23], i.e., all points used in a rule of degree $N$ are included in the set of points for the succeeding rules of degree greater than $N$.

We now consider a family of generalized sequences including (1.6). From now on let $N$ be an even integer of the form $N=2^{n} K \quad(n=1,2, \ldots)$, where $K \in \mathbf{N}$, the set of positive integers, unless otherwise stated. Then, our sequences consist of $3 N, 4 N, 5 N$, that is,

$6 K, 8 K, 10 K, \ldots, 3 \times 2^{n} K, 4 \times 2^{n} K, 5 \times 2^{n} K, \ldots, \quad n=1,2, \ldots, K \in \mathbf{N}$, where the case $K=1$, in particular, coincides with the sequence (1.6). Consequently, the sequences of quadrature rules are of the form $\left\{Q_{3 N}, Q_{4 N}, Q_{5 N}\right\}^{1}$, or equivalently $\left\{Q_{4 N}, Q_{5 N}, Q_{3 \times 2 N}\right\}$.

For integers $m=1,2$, define polynomials $\Omega_{m N}^{ \pm}(x)$ of degree $m N$ by

$$
\Omega_{m N}^{ \pm}(x)=T_{m N}(x)-\cos \{(2 \pm 1) m \pi / 8\}, \quad m=1,2 .
$$

Let $Q_{4 N}(f), Q_{(4+m) N}(f)(m=1,2)$ denote extended CC rules whose abscissae are zeros of $\omega_{4 N+1}(x)$ and of $\omega_{4 N+1}(x) \Omega_{m N}^{+}(x)$, respectively. Then, $Q_{4 N}(f)$ is a CC rule, and for $Q_{(4+m) N}(f)$ we will prove in $\S 2$ the following theorem.

Theorem 1.1. For integers $m=1,2$, let $t_{m N, j}, 0 \leq j \leq m N-1$, be the zeros of $\Omega_{m N}^{+}(x)$ (1.7), that is,

$$
t_{m N, j}=\cos \{2 \pi(j+3 m / 16) /(m N)\}, \quad 0 \leq j \leq m N-1 .
$$

Then, the weights $w_{4 N, i}^{(m)}$ and $u_{m N, j}$ of the extended CC rules $Q_{(4+m) N}(f)$ of degree $(4+m) N, m=1,2$, of closed type, given by

$$
Q_{(4+m) N}(f)=\sum_{i=0}^{4 N} w_{4 N, i}^{(m)} f\left(x_{4 N, i}\right)+\sum_{j=0}^{m N-1} u_{m N, j} f\left(t_{m N, j}\right), \quad m=1,2
$$

\footnotetext{
${ }^{1}$ In the first draft we assumed that $N$ is a power of 2 , but the referee suggested to us that $N$ is allowed to take every even positive integer in proving Theorems 1.1 and 1.2 below.
} 
are all positive, where $x_{4 N, i}=\cos \{\pi i /(4 N)\}, 0 \leq i \leq 4 N$, are the zeros of $\omega_{4 N+1}(x)$ defined by (1.3). For the case $m=2$, the integer $N$ can be allowed to be any positive integer, even or not.

For the expressions of $w_{4 N, i}^{(m)}$ and $u_{m N, j}$, see (2.2) and (2.3), respectively.

Remark. Theorem 1.1 holds only under the assumption that $m N(m=1,2)$ are even; it does not hold when $m N(m=1,2)$ are odd.

The rule $Q_{5 N}(f)$ of degree $5 N$ is embedded in $Q_{6 N}(f)$, which is also an embedded rule in the CC rule $Q_{8 N}(f)$ based on the zeros of $\omega_{8 N+1}(x)$. This is easily seen from the relations (see Lemma 4.1 and (2.4) in [15]):

$$
\begin{aligned}
\omega_{8 N+1}(x) & =2 \omega_{4 N+1}(x) T_{4 N}(x), \\
T_{4 N}(x) & =2 \Omega_{2 N}^{-}(x) \Omega_{2 N}^{+}(x), \\
\Omega_{2 N}^{+}(x) & =2 \Omega_{N}^{+}(x)\left\{T_{N}(x)+\cos 3 \pi / 8\right\} .
\end{aligned}
$$

Omitting the endpoints $x=1$ and $x=-1$ in the $N+1$ abscissae of the CC rule, namely using the zeros of $U_{N-1}(x)$ as the abscissae, Filippi [10] proposed a quadrature rule of open type, having all positive weights. Let $\widetilde{Q}_{4 N-2}(f)$ and $\widetilde{Q}_{(4+m) N-2}(f), m=1,2$, denote extended Filippi rules of open type whose abscissae are the zeros of $U_{4 N-1}(x)$ and those of $U_{4 N-1}(x) \Omega_{m N}^{-}(x)$, respectively. Then $\widetilde{Q}_{4 N-2}(f)$ is the Filippi rule and in $\S 3$ we will prove the following theorem.

Theorem 1.2. For integers $m=1,2$ let $\tau_{m N, j}, 0 \leq j \leq m N-1$, denote the zeros of $\Omega_{m N}^{-}(x)(1.7)$, that is,

$$
\tau_{m N, j}=\cos \{2 \pi(j+m / 16) /(m N)\} .
$$

Then, the weights $v_{4 N, i}^{(m)}$ and $\eta_{m N, j}$ of the extended Filippi rules $\widetilde{Q}_{(4+m) N-2}(f)$ of degree $(4+m) N-2$ of open type, given by

$$
\begin{aligned}
\tilde{Q}_{(4+m) N-2}(f)= & \sum_{i=1}^{4 N-1} v_{4 N, i}^{(m)} f\left(x_{4 N, i}\right) \\
& +\sum_{j=0}^{m N-1} \eta_{m N, j} f\left(\tau_{m N, j}\right), \quad m=1,2,
\end{aligned}
$$

are all positive, where $x_{4 N, i} \quad(1 \leq i \leq 4 N-1)$ are the same as those used in (1.9) except for $x_{4 N, 0}(=1)$ and $x_{4 N, 4 N}(=-1)$. For the case $m=2$, the integer $N$ can be allowed to be any positive integer.

Remark. Theorem 1.2 holds only under the assumption that $m N \quad(m=1,2)$ are even; it does not hold when $m N(m=1,2)$ are odd.

If we note $(1.3),(1.10),(1.11)$, and the relation

$$
\Omega_{2 N}^{-}(x)=2 \Omega_{N}^{-}(x)\left\{T_{N}(x)+\cos \pi / 8\right\},
$$

we can see that $\widetilde{Q}_{5 N-2}(f)$ is an embedded rule in $\widetilde{Q}_{6 N-2}(f)$, which is also embedded in $\widetilde{Q}_{8 N-2}(f)$ based on the $8 N-1$ abscissae, the zeros of $U_{8 N-1}(x)$. 
In $\S 4$ we conclude by suggesting a conjecture on the possibility of some other rules having positive weights.

\section{Proof of Theorem 1.1}

Let $L_{(4+m) N}(f ; x) \quad(m=1,2)$ denote the unique polynomial of degree less than or equal to $(4+m) N$ that coincides with $f$ at the $4 N+1$ points $x_{4 N, i}$, $0 \leq i \leq 4 N$, and $m N$ points $t_{m N, j}, 0 \leq j \leq m N-1$, in (1.8). Then we have the Lagrange interpolation formula for $L_{(4+m) N}(f ; x)$ :

$$
\begin{aligned}
L_{(4+m) N}(f ; x)= & \sum_{i=0}^{4 N} l_{4 N, i}(x) \frac{\Omega_{m N}^{+}(x)}{\Omega_{m N}^{+}\left(x_{4 N, i}\right)} f\left(x_{4 N, i}\right) \\
& +\sum_{j=0}^{m N-1} \frac{\Omega_{m N}^{+}(x)}{\left(x-t_{m N, j}\right) \Omega_{m N}^{+\prime}\left(t_{m N, j}\right)} \\
& \cdot \frac{\omega_{4 N+1}(x)}{\omega_{4 N+1}\left(t_{m N, j}\right)} f\left(t_{m N, j}\right), \quad m=1,2,
\end{aligned}
$$

where $l_{4 N, i}(x)$ is given by (1.5). Integrating $L_{(4+m) N}(f ; x)$ on the range $[-1,1]$ yields the integration rule $Q_{(4+m) N}(f)=\int_{-1}^{1} L_{(4+m) N}(f ; x) d x$ in (1.9), where the weights $w_{4 N, i}^{(m)}$ and $u_{m N, j}$ are given by

$$
\begin{aligned}
& w_{4 N, i}^{(m)}=\int_{-1}^{1} l_{4 N, i}(x) \frac{\Omega_{m N}^{+}(x)}{\Omega_{m N}^{+}\left(x_{4 N, i}\right)} d x, \quad 0 \leq i \leq 4 N, \\
& u_{m N, j}=\int_{-1}^{1} \frac{\Omega_{m N}^{+}(x)}{\left(x-t_{m N, j}\right) \Omega_{m N}^{+\prime}\left(t_{m N, j}\right)} \cdot \frac{\omega_{4 N+1}(x)}{\omega_{4 N+1}\left(t_{m N, j}\right)} d x, \\
& 0 \leq j \leq m N-1, \quad m=1,2 .
\end{aligned}
$$

2.1. Positivity of $w_{4 N, i}^{(m)}$. First, we prove that the weights $w_{4 N, i}^{(m)}, 0 \leq i \leq 4 N$, in (1.9) and given by (2.2) are all positive for the case $m=1$.

Lemma 2.1. Let $l_{4 N, i}(x)$ and $\Omega_{N}^{+}(x)$ be the polynomials defined by (1.5) and (1.7), respectively. Then we have

$$
\begin{aligned}
& 4 N l_{4 N, i}(x) \Omega_{N}^{+}(x)= 2 \Omega_{N}^{+}\left(x_{4 N, i}\right) \sum_{k=0}^{4 N} T_{k}\left(x_{4 N, i}\right) T_{k}(x) \\
&+\sum_{k=0}^{N} T_{3 N+k}\left(x_{4 N, i}\right)\left\{T_{4 N+k}(x)-T_{4 N-k}(x)\right\}, \\
& 0 \leq i \leq 4 N,
\end{aligned}
$$

where $x_{4 N, i}$ are the zeros of $\omega_{4 N+1}(x)(1.3)$.

Proof. Using the relation

$$
2 T_{k}(x) T_{n}(x)=T_{k+n}(x)+T_{|k-n|}(x), \quad k, n \geq 0,
$$


from (1.5) and (1.7) we have

$$
\begin{aligned}
4 N & l_{4 N, i}(x) \Omega_{N}^{+}(x) \\
= & 2 \sum_{k=0}^{4 N} T_{k}\left(x_{4 N, i}\right) T_{k}(x)\left\{T_{N}(x)-\cos 3 \pi / 8\right\} \\
= & \sum_{k=0}^{4 N} T_{k}\left(x_{4 N, i}\right)\left\{T_{N+k}(x)+T_{|N-k|}(x)-2 \cos (3 \pi / 8) T_{k}(x)\right\} \\
= & \sum_{k=0}^{4 N}\left\{T_{N+k}\left(x_{4 N, i}\right)+T_{|N-k|}\left(x_{4 N, i}\right)-2 \cos (3 \pi / 8) T_{k}\left(x_{4 N, i}\right)\right\} T_{k}(x) \\
& +\sum_{k=0}^{N}\left\{T_{3 N+k}\left(x_{4 N, i}\right) T_{4 N+k}(x)-T_{4 N+k}\left(x_{4 N, i}\right) T_{3 N+k}(x)\right\} .
\end{aligned}
$$

If we again use (2.5) on the far right of (2.6) and note that

$$
T_{8 N-k}\left(x_{4 N, i}\right)=T_{k}\left(x_{4 N, i}\right), \quad 0 \leq k \leq 8 N,
$$

it is easy to verify $(2.4)$.

Substituting (2.4) into (2.2) yields the expression for the weights $w_{4 N, i}^{(1)}$ :

$$
4 N w_{4 N, i}^{(1)}=2 \sum_{k=0}^{4 N} T_{k}\left(x_{4 N, i}\right) \mu(k)+\sum_{k=0}^{N} \frac{T_{3 N+k}\left(x_{4 N, i}\right)}{\Omega_{N}^{+}\left(x_{4 N, i}\right)} \cdot \phi_{N}(k),
$$

where $\mu(k)$ and $\phi_{N}(k)$ are defined by

$$
\begin{gathered}
\mu(k)=\int_{-1}^{1} T_{k}(x) d x= \begin{cases}2 /\left(1-k^{2}\right) & \text { if } k=\text { even }, \\
0 & \text { if } k=\text { odd },\end{cases} \\
\phi_{N}(k)=\mu(4 N+k)-\mu(4 N-k), \quad k \geq 0 .
\end{gathered}
$$

For real $x \geq 2$, let $\mu(x)$ be defined by

$$
\mu(x)=2 /\left(1-x^{2}\right), \quad x \geq 2,
$$

instead of (2.9) and let $\phi_{N}(x)$ be defined by (2.10) with $\mu(x)$ given by (2.11). Then $\mu(x)$ is negative and monotone increasing for $x \geq 2$ and $\phi_{N}(x)$ is also a monotone increasing function of $x$ when $0 \leq x \leq N$. We will take advantage of these properties in proving the positivity of weights of our extended $\mathrm{CC}$ rules.

Lemma 2.2. Let $x_{4 N, i}, 0 \leq i \leq 4 N$, be the zeros of $\omega_{4 N+1}(x)(1.3)$ and $\Omega_{N}^{+}(x)$ be defined by (1.7). Then we have

$$
\begin{aligned}
\left\{2 \Omega_{N}^{+}\left(x_{4 N, i}\right)\right\}^{-1}= & T_{N}\left(x_{4 N, i}\right)+2 \cos (3 \pi / 8) T_{2 N}\left(x_{4 N, i}\right) \\
& +(1+2 \cos 3 \pi / 4) T_{3 N}\left(x_{4 N, i}\right) \\
& +2 \cos (3 \pi / 4) \cos (3 \pi / 8) T_{4 N}\left(x_{4 N, i}\right),
\end{aligned}
$$

$$
0 \leq i \leq 4 N \text {. }
$$

Proof. From (1.11) and (1.12) and the relation $\left\{T_{4 N}\left(x_{4 N, i}\right)\right\}^{2}=1$ for $0 \leq i \leq$ $4 N$, it follows that

$$
1 / \Omega_{N}^{+}\left(x_{4 N, i}\right)=4\left\{T_{N}\left(x_{4 N, i}\right)+\cos 3 \pi / 8\right\} \Omega_{2 N}^{-}\left(x_{4 N, i}\right) T_{4 N}\left(x_{4 N, i}\right) .
$$


In (2.13), using (2.5), (2.7) and the relation $\Omega_{2 N}^{-}(x)=T_{2 N}(x)+\cos (3 \pi / 4)$ (see (1.7)) establishes (2.12).

Theorem 2.3. Let $w_{4 N, i}^{(1)}, 0 \leq i \leq 4 N$, denote the weights given by (1.9) and $\mu(k)$ and $\phi_{N}(k)$ be defined by (2.9) and (2.10), respectively. Define $A_{N l}(k)$, $0 \leq l \leq 3, b y$

$$
\begin{gathered}
A_{N 0}(k)=2 \mu(k)+(1+2 \cos 3 \pi / 4) \phi_{N}(k) \\
+4 \cos (3 \pi / 4) \cos (3 \pi / 8) \phi_{N}(N-k), \\
A_{N 1}(k)=2 \mu(N+k)+2 \cos (3 \pi / 8) \phi_{N}(k) \\
+(1+2 \cos 3 \pi / 4) \phi_{N}(N-k), \\
A_{N 2}(k)=2 \mu(2 N+k)+\phi_{N}(k)+2 \cos (3 \pi / 8) \phi_{N}(N-k), \\
A_{N 3}(k)=2 \mu(3 N+k)+\phi_{N}(N-k), \quad 0 \leq k \leq N .
\end{gathered}
$$

Then we have

$$
w_{4 N, i}^{(1)}=\frac{1}{4 N} \sum_{k=0}^{N} \sum_{l=0}^{3} A_{N l}(k) T_{l N+k}\left(x_{4 N, i}\right), \quad 0 \leq i \leq 4 N,
$$

and $A_{N l}(k), 0 \leq l \leq 3$, are all negative except for $A_{N 0}(0)$.

Proof. Using (2.5), (2.7) and (2.12) in (2.8) establishes (2.18). We make use of the fact that $\mu(k)<0$ for $k>0$ and $\phi_{N}(k) \geq 0 \quad(0 \leq k \leq N)$ to show that $A_{N l}(k) \quad(0 \leq l \leq 3,0 \leq k \leq N)$ are negative except for $A_{N 0}(0)$. Noting that the coefficients of $\phi_{N}(k)$ and $\phi_{N}(N-k)$ in (2.14) are both negative, we can see that $A_{N 0}(k)<0 \quad(0<k \leq N)$. It can be easily seen from (2.10) and (2.17) that $A_{N 3}(k)=\mu(3 N+k)+\mu(5 N-k)<0 \quad(0 \leq k \leq N)$. If we note in (2.15) that $\mu(N+k)-\cos (3 \pi / 8) \mu(4 N-k)$ is negative for $0 \leq k \leq N$, we can see that $A_{N 1}(k)<0 \quad(0 \leq k \leq N)$. To verify that $A_{N 2}(k)<0$, it suffices to use in (2.16) the definitions (2.9) and (2.10) and the relation

$$
\begin{aligned}
& 2 \mu(2 N+k)-2 \cos (3 \pi / 8) \mu(3 N+k)-\mu(4 N-k) \\
& \quad \leq\{2-2 \cos (3 \pi / 8)-1\} \mu(3 N)<0, \quad 0 \leq k \leq N .
\end{aligned}
$$

Noting that $T_{l}\left(x_{4 N, i}\right)<1,1 \leq i \leq 4 N-1$, and $T_{l}\left(x_{4 N, 0}\right)=1$, we have from Theorem 2.3 that

$$
\begin{aligned}
4 N w_{4 N, i}^{(1)} & \geq \sum_{k=0}^{N} \sum_{l=0}^{3} A_{N l}(k) \\
& =2 \sum_{k=0}^{4 N} \mu(k)+4\left(1+\cos \frac{3 \pi}{4}\right)\left(1+\cos \frac{3 \pi}{8}\right) \sum_{k=0}^{N} \phi_{N}^{\prime \prime}(k)>0,
\end{aligned}
$$

where, in the last inequality above, we have used the relation

$$
\sum_{k=0}^{4 N} \mu(k)=4 N /\left\{(4 N)^{2}-1\right\}>0 .
$$

Thus, $w_{4 N, i}^{(1)}, 0 \leq i \leq 4 N$, have been verified to be all positive. Verification of the positivity of $w_{4 N, i}^{(2)}(0 \leq i \leq 4 N)$ can be accomplished similarly, in fact more easily, but we omit the details. 
2.2. Positivity of $u_{m N, j}$. We begin by proving that the weights $u_{m N, j}, 0 \leq$ $j \leq N-1$, in (1.9) and given by (2.3) are all positive when $m=1$. It is similar and more easy to prove the positivity of $u_{2 N, j}, 0 \leq j \leq 2 N-1$, but we omit the details.

Lemma 2.4. Let $\Omega_{N}^{+}(x)$ be the polynomial defined by (1.7) and $t_{N j}, 0 \leq j \leq$ $N-1$, be the zeros of $\Omega_{N}^{+}(x)$, given by (1.8). Then we have

$$
\frac{\Omega_{N}^{+}(x)}{x-t_{N j}}=2 \sum_{k=0}^{N} T_{N-k}\left(t_{N j}\right) U_{k-1}(x),
$$

where we define $U_{-1}(x)=0$.

Proof. From the assumption, it follows that

$$
\frac{\Omega_{N}^{+}(x)}{x-t_{N j}}=\frac{\Omega_{N}^{+}(x)-\Omega_{N}^{+}\left(t_{N j}\right)}{x-t_{N j}}=\frac{T_{N}(x)-T_{N}\left(t_{N j}\right)}{x-t_{N j}} .
$$

Elliott [8] gives the identity

$$
T_{n}(x)-T_{n}(y)=2(x-y) \sum_{k=0}^{n} T_{n-k}(y) U_{k-1}(x), \quad n \geq 0 .
$$

Using (2.22) in (2.21) establishes (2.20).

Lemma 2.5. For the weights $u_{N j}, 0 \leq j<N$, given by (1.9) and (2.3), we have

$$
\begin{aligned}
N \sin (3 \pi / 8) u_{N j} & =\sum_{k=0}^{N} T_{N-k}\left(t_{N j}\right) \phi_{N}(k) \\
& =\sum_{k=0}^{N / 2} \cos \left(2 k \xi_{N j}\right) \phi_{N}(N-2 k),
\end{aligned}
$$

where $\phi_{N}(k)$ is given by (2.10) and $\xi_{N j}$ is defined by

$$
t_{N j}=\cos \xi_{N j}, \quad \xi_{N j}=2 \pi(j+3 / 16) / N, \quad 0 \leq j \leq N-1 .
$$

Proof. From (1.3) and the definition of $U_{k}(x)$ it follows that

$$
\omega_{4 N+1}(x) U_{k-1}(x)=T_{4 N+k}(x)-T_{|4 N-k|}(x) .
$$

From (1.8), (2.25) and the relation

$$
\Omega_{N}^{ \pm \prime}(x)=N U_{N-1}(x)
$$

we have

$$
\begin{aligned}
\Omega_{N}^{+\prime}\left(t_{N j}\right) \omega_{4 N+1}\left(t_{N j}\right) & =N\left\{T_{5 N}\left(t_{N j}\right)-T_{3 N}\left(t_{N j}\right)\right\} \\
& =-2 N \sin \frac{3 \pi}{2} \sin \frac{3 \pi}{8}=2 N \sin \frac{3 \pi}{8} .
\end{aligned}
$$

Using (2.20), (2.25) and (2.27) in (2.3) and noting (2.9) and (2.10) establishes (2.23).

The Poisson Summation formula [17, p. 270], [19] is helpful in proving that $u_{N j}>0$ when $0 \leq j<N$. 
Lemma 2.6 (Poisson summation formula). Let $\psi(x)$ be an integrable function defined on the real positive line and $\Psi(\omega)$ be the Fourier cosine transform of $\psi(x)$,

$$
\Psi(\omega)=\int_{0}^{\infty} \psi(x) \cos \omega x d x .
$$

Then for any positive $\zeta$ and real $x$ we have the formula

$$
\sum_{k=0}^{\infty}\{\Psi(k \zeta+x)+\Psi(k \zeta-x)\}=\frac{2 \pi}{\zeta} \sum_{k=0}^{\prime} \psi\left(\frac{2 \pi k}{\zeta}\right) \cos \left(\frac{2 \pi k x}{\zeta}\right),
$$

where the prime denotes the summation whose first term is halved.

Define $\psi(x)$ by

$$
\psi(x)=\phi_{N}(N-2 x)=2 /\left\{1-(5 N-2 x)^{2}\right\}-2 /\left\{1-(3 N+2 x)^{2}\right\}
$$

for $0 \leq x \leq N / 2$, and by $\psi(x)=0$ otherwise. Then, making use of the formula (2.29) in (2.23), we have

$$
\begin{array}{r}
N \sin (3 \pi / 8) u_{N j}=\sum_{k=0}^{N / 2} \cos \left(2 k \xi_{N j}\right) \psi(k)=\sum_{k=0}^{\infty} \cos \left(2 k \xi_{N j}\right) \psi(k) \\
=\sum_{k=0}^{\infty}\left\{\Phi\left(2 \pi k+2 \xi_{N j}\right)+\Phi\left(2 \pi k-2 \xi_{N j}\right)\right\}, \\
0 \leq j \leq N-1,
\end{array}
$$

where $\Phi(\omega)$ is given by

$$
\Phi(\omega)=\int_{0}^{N / 2} \phi_{N}(N-2 x) \cos \omega x d x .
$$

In order to prove that $u_{N j}>0$ in (2.31), it suffices to show that $\Phi(\omega)>0$ for any real $\omega$. From (2.30), $\phi_{N}(x)$ is found to be an odd function and can be expanded as follows:

$$
\begin{aligned}
\phi_{N}(x) & =2 /\left\{1-(4 N+x)^{2}\right\}-2 /\left\{1-(4 N-x)^{2}\right\} \\
& =2 x /\left\{(4 N-1)^{2}-x^{2}\right\}-2 x /\left\{(4 N+1)^{2}-x^{2}\right\} \\
& =2 \sum_{l=1}^{\infty} c_{l} x^{l}, \quad 0 \leq x \leq N,
\end{aligned}
$$

where $c_{l}$ for odd $l$ is defined by

$$
c_{l}=(4 N-1)^{-l-1}-(4 N+1)^{-l-1}>0,
$$

and $c_{l}$ vanishes when $l$ is an even integer. Substituting the expansion (2.33) into the right-hand side of (2.32) gives

$$
\Phi(\omega)=2 \sum_{l=1}^{\infty} c_{l} \mathbf{\Theta}_{l}(\omega),
$$

where $\Theta_{l}(\omega)$ is defined by

$$
\boldsymbol{\Theta}_{l}(\omega)=\int_{0}^{N / 2}(N-2 x)^{l} \cos \omega x d x, \quad l \geq 1 .
$$


It remains to show that $\theta_{l}(\omega)$ is positive, since then $\Phi(\omega)$ is positive by $(2.34)$ and (2.35).

To this end, we define $\rho(x)=N-2|x|$ when $|x| \leq N / 2$, and $\rho(x)=0$ otherwise. Then $\boldsymbol{\theta}_{l}(\omega)$ (2.36) can be rewritten as

$$
\begin{aligned}
\boldsymbol{\Theta}_{l}(\omega) & =\int_{-\infty}^{\infty}\{\rho(x)\}^{l} e^{i \omega x} d x \\
& =\pi^{1-l} \overbrace{\boldsymbol{\theta}_{1} * \boldsymbol{\theta}_{1} * \cdots * \boldsymbol{\theta}_{1}(\omega)}^{l}, \quad l \geq 1,
\end{aligned}
$$

where $g * h(\omega)$ denotes convolution defined by

$$
g * h(\omega)=\int_{-\infty}^{\infty} g(\omega-t) h(t) d t
$$

Since $\boldsymbol{\theta}_{1}(\omega)=2\{1-\cos (\omega N / 2)\} / \omega^{2} \geq 0$, it follows from $(2.37)$ that $\boldsymbol{\theta}_{l}(\omega)>0$ for $l \geq 2$.

\section{Proof OF TheOREM 1.2}

Let $h_{N i}(x)$ be defined by

$$
h_{N i}(x)=\frac{U_{N-1}(x)}{\left(x-x_{N i}\right) U_{N-1}^{\prime}\left(x_{N i}\right)}, \quad 1 \leq i \leq N-1,
$$

where $x_{N i}, 1 \leq i \leq N-1$, are the zeros of $U_{N-1}(x) \quad\left(=2 \omega_{N+1}(x) /\left(x^{2}-1\right)\right)$. Then for the weights $v_{4 N, i}^{(m)}$ and $\eta_{m N, j}$ of the quadrature rules $\widetilde{Q}_{(4+m) N-2}(f)$ (1.14), we have, similarly to (2.2) and (2.3),

$$
\begin{gathered}
v_{4 N, i}^{(m)}=\int_{-1}^{1} h_{4 N, i}(x) \frac{\Omega_{m N}^{-}(x)}{\Omega_{m N}^{-}\left(x_{4 N, i}\right)} d x, \quad 1 \leq i \leq 4 N-1, \\
\eta_{m N, j}=\int_{-1}^{1} \frac{\Omega_{m N}^{-}(x)}{\left(x-\tau_{m N, j}\right) \Omega_{m N}^{-\prime}\left(\tau_{m N, j}\right)} \cdot \frac{U_{4 N-1}(x)}{U_{4 N-1}\left(\tau_{m N, j}\right)} d x, \\
0 \leq j \leq m N-1, \quad m=1,2 .
\end{gathered}
$$

We remark that by using $h_{N i}(x)(3.1)$, the Filippi rule [10] $\widetilde{Q}_{N-2}(f)$ based on the points $x_{N i}, 1 \leq i \leq N-1$, can be written as

$$
\widetilde{Q}_{N-2}(f)=\sum_{i=1}^{N-1} \int_{-1}^{1} h_{N i}(x) d x f\left(x_{N i}\right) .
$$

3.1. Positivity of $v_{4 N, i}^{(m)}$. Here we prove that $v_{4 N, i}^{(1)}, 1 \leq i \leq 4 N-1$ (3.2), are all positive. The positivity of $v_{4 N, i}^{(2)}, 1 \leq i \leq 4 N-1$, is more easily verified, but we omit the details.

Filippi (see [10, equation (11)]) shows that $h_{4 N, i}(x)$ in (3.1) and (3.4) can be expressed as follows:

$$
\begin{aligned}
4 N h_{4 N, i}(x) & =2\left(1-x_{4 N, i}^{2}\right) \sum_{k=0}^{4 N-2} U_{k}\left(x_{4 N, i}\right) U_{k}(x) \\
& =\sum_{k=1}^{4 N-1}\left\{T_{k-1}\left(x_{4 N, i}\right)-T_{k+1}\left(x_{4 N, i}\right)\right\} U_{k-1}(x) .
\end{aligned}
$$


Using (2.7) and the relation

$$
U_{k}(x)-U_{k-2}(x)=2 T_{k}(x),
$$

where we define $U_{-k}(x)=-U_{k-2}(x)$, and $T_{-k}(x)=T_{k}(x)$ for $k \geq 0$, we have from (3.5)

$$
\begin{aligned}
4 N h_{4 N, i}(x)= & 2 \sum_{k=0}^{4 N} T_{k}\left(x_{4 N, i}\right) T_{k}(x)-T_{4 N-1}\left(x_{4 N, i}\right) U_{4 N-1}(x) \\
& -T_{4 N}\left(x_{4 N, i}\right)\left\{U_{4 N}(x)+U_{4 N-2}(x)\right\} / 2 .
\end{aligned}
$$

Further, similarly as in the proof of Lemma 2.1 , we have

$$
\begin{aligned}
& 2 \Omega_{N}^{-}(x) \sum_{k=0}^{4 N} T_{k}\left(x_{4 N, i}\right) T_{k}(x) \\
&= 2 \Omega_{N}^{-}\left(x_{4 N, i}\right) \sum_{k=0}^{4 N} T_{k}\left(x_{4 N, i}\right) T_{k}(x) \\
&+\sum_{k=0}^{N} T_{3 N+k}\left(x_{4 N, i}\right)\left\{T_{4 N+k}(x)-T_{4 N-k}(x)\right\}, \\
& 1 \leq i \leq 4 N-1 .
\end{aligned}
$$

Lemma 3.1. For the weights $v_{4 N, i}^{(1)}$ given by (3.2) of the quadrature rule (1.14), we have

$$
\begin{aligned}
4 N v_{4 N, i}^{(1)}= & 2 \sum_{k=0}^{4 N} T_{k}\left(x_{4 N, i}\right) \mu(k)+\sum_{k=0}^{N} \frac{T_{3 N+k}\left(x_{4 N, i}\right)}{\Omega_{N}^{-}\left(x_{4 N, i}\right)} \cdot \phi_{N}(k) \\
& -\frac{T_{4 N}\left(x_{4 N, i}\right)}{\Omega_{N}^{-}\left(x_{4 N, i}\right)} \cdot B_{N}
\end{aligned}
$$

where $\mu(k)$ and $\phi_{N}(k)$ are defined by (2.9) and (2.10), respectively. In (3.9), $B_{N}$ is a constant defined by

$$
B_{N}=\{\nu(5 N)+\nu(3 N)-2 \cos (\pi / 8) \nu(4 N)\} / 2,
$$

and $\nu(n)$ is defined by

$$
\begin{aligned}
\nu(n) & =\frac{1}{2} \int_{-1}^{1}\left\{U_{n}(x)+U_{n-2}(x)\right\} d x \\
& = \begin{cases}1 /(n+1)+1 /(n-1) & \text { if } n=\text { even }, \\
0 & \text { if } n=\text { odd. }\end{cases}
\end{aligned}
$$

Proof. Using (1.7), (3.7) and (3.8) in (3.2) and noting that $\int_{-1}^{1} U_{k}(x) d x=0$ for $k$ odd, and

$$
2 T_{l}(x) U_{m}(x)=U_{l+m}(x)+U_{m-l}(x),
$$

we can easily verify (3.9).

We remark that $B_{N}$ in (3.10) is positive. The following lemma is established along the lines of the proof of Lemma 2.2. 
Lemma 3.2. Let $x_{4 N, i}, 1 \leq i \leq 4 N-1$, be the zeros of $U_{4 N-1}(x)$ and $\Omega_{N}^{-}(x)$ be defined by (1.7). Then we have

$$
\begin{aligned}
\left\{2 \Omega_{N}^{-}\left(x_{4 N, i}\right)\right\}^{-1}= & T_{N}\left(x_{4 N, i}\right)+2 \cos (\pi / 8) T_{2 N}\left(x_{4 N, i}\right) \\
& +(1+2 \cos \pi / 4) T_{3 N}\left(x_{4 N, i}\right) \\
& +2 \cos (\pi / 4) \cos (\pi / 8) T_{4 N}\left(x_{4 N, i}\right), \\
& 1 \leq i \leq 4 N-1 .
\end{aligned}
$$

Theorem 3.3. Let $v_{4 N, i}^{(1)}, \quad 1 \leq i \leq 4 N-1$, denote the weights in (1.14) and $\mu(k), \phi_{N}(k)$ and the constant $B_{N}$ be defined by (2.9), (2.10), and (3.10), respectively. Let $A_{N l}(k), 0 \leq l \leq 3$, denote the constants given by (2.14)-(2.17) with $\cos 3 \pi / 4$ and $\cos 3 \pi / 8$ replaced by $\cos \pi / 4$ and $\cos \pi / 8$, respectively. Then we have

$$
\begin{aligned}
& v_{4 N, i}^{(1)}= \frac{1}{4 N} \sum_{k=0}^{N} \sum_{l=0}^{\prime \prime} A_{N l}(k) T_{l N+k}\left(x_{4 N, i}\right) \\
&-\frac{1}{4 N} \cdot \frac{T_{4 N}\left(x_{4 N, i}\right) B_{N}}{\Omega_{N}^{-}\left(x_{4 N, i}\right)}, \quad 0 \leq i \leq 4 N,
\end{aligned}
$$

and $A_{N l}(k), 0 \leq l \leq 3$, are all negative except for $A_{N 0}(0)$.

Proof. Verification of (3.14) is accomplished along the lines of the proof of Theorem 2.3. We have already verified in the proof of Theorem 2.3 that $A_{N 3}(k)<0$. We make use of the fact that $\mu(k)$ and $\phi_{N}(k)$ vanish when $k$ is odd and are monotone increasing functions for even integers $k$ satisfying $2 \leq k \leq N$ to show that

$$
\begin{aligned}
A_{N 0}(k) & =2 \mu(k)+(1+2 \cos \pi / 4) \phi_{N}(k)+4 \cos (\pi / 4) \cos (\pi / 8) \phi_{N}(N-k) \\
& \leq 2 \mu(N)+c \phi_{N}(N) \\
& =2 \mu(N)-c \mu(3 N)+c \mu(5 N), \quad 2 \leq k \leq N,
\end{aligned}
$$

where we put $c=1+2 \cos (\pi / 4)(1+2 \cos \pi / 8)=5.027 \ldots$ It is easy to verify that $2 \mu(N)-c \mu(3 N)<0$ for $N \geq 2$. Thus, it is seen that $A_{N 0}(k)<0$ for $2 \leq k \leq N$ because $\mu(k)<0$ for $k \geq 2$. Similarly, we have

$$
\begin{aligned}
A_{N 1}(k) & =2 \mu(N+k)+2 \cos (\pi / 8) \phi_{N}(k)+(1+2 \cos \pi / 4) \phi_{N}(N-k) \\
& \leq 2 \mu(2 N)+b \phi_{N}(N)<0, \quad 0 \leq k \leq N,
\end{aligned}
$$

where we have put $b=1+2(\cos \pi / 4+\cos \pi / 8)=4.261 \ldots$.

Next, we show that $A_{N 2}(k)<0$ for $0 \leq k \leq N$. We can write $A_{N 2}(k)$ in the form

$$
\begin{aligned}
A_{N 2}(k)= & 2 \mu(2 N+k)+2 \cos (\pi / 8) \phi_{N}(N-k)+\phi_{N}(k) \\
= & 2\{\mu(2 N+k)-\cos (\pi / 8) \mu(3 N+k)\}+\mu(4 N+k) \\
& +\{2 \cos (\pi / 8) \mu(5 N-k)-\mu(4 N-k)\} .
\end{aligned}
$$

It is trivial that the first and second terms on the far right of (3.15) are negative. Close inspection reveals that the third term is also negative. Thus we have that $A_{N 2}(k)<0$. 
Noting that $T_{l}\left(x_{4 N, i}\right)<1,1 \leq i \leq 4 N-1$, and $T_{l}\left(x_{4 N, 0}\right)=1$, from (3.13) and Theorem 3.3 we have

$$
\begin{aligned}
4 N v_{4 N, i}^{(1)} & >\sum_{k=0}^{N} \sum_{l=0}^{3} A_{N l}(k)-4 B_{N}\left(1+\cos \frac{\pi}{4}\right)\left(1+\cos \frac{\pi}{8}\right) \\
& =2 \sum_{k=0}^{4 N} \mu(k)+4\left(1+\cos \frac{\pi}{4}\right)\left(1+\cos \frac{\pi}{8}\right)\left\{\sum_{k=0}^{N \prime \prime} \phi_{N}(k)-B_{N}\right\} .
\end{aligned}
$$

Using the relation (2.19), that is,

$$
2 \sum_{k=0}^{4 N} \mu(k)=\nu(4 N)
$$

and

$$
2 \sum_{k=0}^{N} \phi_{N}(k)=\nu(3 N)+\nu(5 N)-2 \nu(4 N),
$$

we have from (3.10) and (3.16)

$$
4 N v_{4 N, i}^{(1)}>\nu(4 N)+4\left(1+\cos \frac{\pi}{4}\right)\left(1+\cos \frac{\pi}{8}\right)\left(\cos \frac{\pi}{8}-1\right) \nu(4 N)=0 .
$$

Thus, the weights $v_{4 N, i}^{(1)} 1 \leq i \leq 4 N-1$, are all positive.

3.2. Positivity of $\eta_{m N, j}$. We prove that the weights $\eta_{N j}, 0 \leq j \leq N-1$, in (1.14) are all positive. It is similar and more easy to verify the positivity of $\eta_{2 N, j}(0 \leq j \leq 2 N-1)$, but we omit details.

Lemma 3.4. Let $\Omega_{N}^{-}(x)$ be the polynomial defined by (1.7) and $\tau_{N j}, 0 \leq j \leq$ $N-1$, be the zeros of $\Omega_{N}^{-}(x)$ given by (1.13). Then we have

$$
\frac{\Omega_{N}^{-}(x)}{x-\tau_{N j}}=2 \sum_{k=0}^{N} U_{N-1-k}\left(\tau_{N j}\right) T_{k}(x) .
$$

Proof. Identity (3.19) is established along the lines of the proof of Lemma 2.4.

Lemma 3.5. For the weights $\eta_{N j}$ in (1.14) and (3.3), we have

$$
N \sin (\pi / 8) \eta_{N j}=B_{N}-\sum_{k=0}^{N} T_{N-k}\left(\tau_{N j}\right) \phi_{N}(k), \quad 0 \leq j \leq N-1,
$$

where $B_{N}$ is given by (3.10). 
Proof. Using (3.12) and (3.19), we have

$$
\begin{aligned}
2\left(\tau_{N j}^{2}-1\right) & \frac{U_{4 N-1}(x) \Omega_{N}^{-}(x)}{x-\tau_{N j}} \\
= & \sum_{k=0}^{N}\left\{T_{N+1-k}\left(\tau_{N j}\right)-T_{N-1-k}\left(\tau_{N j}\right)\right\}\left\{U_{4 N+k-1}(x)+U_{4 N-k-1}(x)\right\} \\
= & \sum_{k=-1}^{N-1} T_{N-k}\left(\tau_{N j}\right)\left\{U_{4 N+k}(x)+U_{4 N-k-2}(x)\right\} \\
& -\sum_{k=1}^{N+1} T_{N-k}\left(\tau_{N j}\right)\left\{U_{4 N-k}(x)+U_{4 N+k-2}(x)\right\} .
\end{aligned}
$$

On the other hand, using (1.13), (2.25) and (2.26), we have

$$
\begin{gathered}
2\left(\tau_{N j}^{2}-1\right) U_{4 N-1}\left(\tau_{N j}\right) \Omega_{N}^{-1}\left(\tau_{N j}\right)=N \omega_{4 N+1}\left(\tau_{N j}\right) U_{N-1}\left(\tau_{N j}\right) \\
=N\left\{T_{5 N}\left(\tau_{N j}\right)-T_{3 N}\left(\tau_{N j}\right)\right\}=-2 N \sin (\pi / 8) .
\end{gathered}
$$

Substituting (3.21) and (3.22) into (3.3) and using (3.6) yields (3.20). have

From (3.10), (3.17) and (3.20) and the fact that the $\phi_{N}(k)$ are positive, we

$$
\begin{aligned}
N \sin (\pi / 8) \eta_{N j} & \geq B_{N}-\sum_{k=0}^{N} \phi_{N}(k) \\
& =2\{1-\cos (\pi / 8)\} \nu(4 N)>0, \quad 0 \leq j \leq N-1 .
\end{aligned}
$$

Thus, the weights $\eta_{N j}, 0 \leq j \leq N-1$, are all positive.

\section{CONJECTURE ON THE POSSIBILITY OF OTHER POSITIVE QUADRATURE RULES}

Before we give some conjectures on the possibility of other extended CC rules having all positive weights, we briefly review the sequence of uniform distribution on $(0,1)[15,16]$.

Let any positive integer $l$ be written in radix-2 notation as

$$
l=l_{m} l_{m-1} \cdots l_{2} l_{1}=l_{1}+l_{2} \cdot 2+\cdots+l_{m} \cdot 2^{m-1},
$$

where $m=\left[\log _{2} l\right]+1, l_{i}=0$ or $1(1 \leq i \leq m-1)$, and always $l_{m}=1$. Define fractions $\beta_{l}$ and $\alpha_{l}$ by

$$
\beta_{l}=l_{1} \cdot 2^{-1}+l_{2} \cdot 2^{-2}+\cdots+l_{m-1} \cdot 2^{-m+1}+l_{m} \cdot 2^{-m}+1 \cdot 2^{-m-1}
$$

and $\alpha_{l}=\beta_{l}-2^{-m}$. Then $\beta_{l}$ (and $\alpha_{l}$ ) can be seen to satisfy the recurrence relations

$$
\beta_{2 l}=\beta_{l} / 2, \quad \beta_{2 l+1}=\beta_{2 l}+1 / 2, \quad l \geq 1,
$$

with the starting value $\beta_{1}=3 / 4$ (and $\alpha_{1}=1 / 4$ ).

The sequence $\left\{\beta_{l}-2^{-1-m}\right\}$ is the so-called Van der Corput sequence [15], which is uniformly distributed on $(0,1)$, so that the sequence $\left\{\beta_{l}\right\}$ and $\left\{\alpha_{l}\right\}$ are also uniformly distributed on $(0,1)$.

Defining $\beta_{-1}=0$ and $\beta_{0}=1 / 2$, we use two sets, $\left\{\cos 2 \pi \beta_{i}\right\} \quad(-1 \leq i<l)$ and $\left\{\cos 2 \pi \alpha_{i}\right\} \quad(1 \leq i<l)$, as the sample points for interpolatory integration rules of closed type and open type, respectively. 
Lemma 4.1. Let $N$ be a power of $2, N=2^{n}$, and put $x_{k}=\cos 2 \pi \alpha_{k}$ (or $\left.\cos 2 \pi \beta_{k}\right)$. Then for a positive integer $l$ we have

$$
\begin{aligned}
& 2^{N-1} \prod_{k=1}^{N-1}\left(x-x_{k}\right)=U_{N-1}(x), \quad \beta_{N-1}=1-\alpha_{N / 2}, \\
& 2^{N-1} \prod_{k=0}^{N-1}\left(x-x_{l N+k}\right)=T_{N}(x)-x_{l}, \quad \alpha_{N-1}=1-\beta_{N / 2} .
\end{aligned}
$$

Corollary. For $N=2^{n}, n=1,2, \ldots$, we have

$$
2^{N} \prod_{k=-1}^{N-1}\left(x-\cos 2 \pi \beta_{k}\right)=T_{N+1}(x)-T_{N-1}(x)=\omega_{N+1}(x) .
$$

Remark. Lemma 4.1 and its Corollary indicate that the set of the first $2^{n}+1$ $\left(2^{n}-1\right)$ sample points $\left\{\cos 2 \pi \beta_{l}\right\},-1 \leq l \leq 2^{n}-1 \quad\left(\left\{\cos 2 \pi \alpha_{l}\right\}, 1 \leq l \leq\right.$ $\left.2^{n}-1\right)$, coincides with that used in the CC rule (Filippi rule).

Numerical tests suggest the following conjectures.

Conjecture 1. Let the integers $N$ and $M$ be powers of 2 such that $N=2^{n}$ and $M=2^{m}$, where $1 \leq m \leq n-1$ for $n \geq 2$. Then the interpolatory integration rules of degree $N+M$ of closed type based on the set of sample points $\left\{\cos 2 \pi \beta_{l}\right\}$, $-1 \leq l \leq N+M-1$, have all positive weights.

We note $[15,16]$ that the set of the first $N+M+1$ sample points $\left\{\cos 2 \pi \beta_{l}\right\}$, $-1 \leq l \leq N+M-1$, coincides with the zeros of the polynomial

$$
\left\{T_{N+1}(x)-T_{N-1}(x)\right\}\left\{T_{M}(x)-\cos 3 \pi M /(2 N)\right\} .
$$

In $\S 2$ we have verified special cases of Conjecture 1 , namely the cases $M=N / 2$ and $M=N / 4$.

Conjecture 2. Let the integers $N$ and $M$ be powers of 2 such that $N=2^{n}$ and $M=2^{m}$, where $1 \leq m \leq n-2$ for $n \geq 3$. Then the interpolatory integration rules of degree $N-M$ of closed type based on the set of sample points $\left\{\cos 2 \pi \beta_{l}\right\}$, $-1 \leq l \leq N-M-1$, have all positive weights.

Remark. The set of sample points $\left\{\cos 2 \pi \beta_{l}\right\},-1 \leq l \leq N-M-1$, coincides with the zeros of the polynomial

$$
\left\{T_{N+1}(x)-T_{N-1}(x)\right\} /\left\{T_{M}(x)-\cos \pi M / N\right\} .
$$

Conjecture 3. Under the same assumption as in Conjecture 1, the interpolatory integration rules of degree $N+M-2$ of open type based on the set of sample points $\left\{\cos 2 \pi \alpha_{l}\right\}, 1 \leq l \leq N+M-1$, have all positive weights.

Remark. The set of sample points $\left\{\cos 2 \pi \alpha_{l}\right\}, 1 \leq l \leq N+M-1$, coincides with the zeros of the polynomial

$$
U_{N-1}(x)\left\{T_{M}(x)-\cos \pi M /(2 N)\right\} .
$$

In $\S 3$ we have verified special cases of Conjecture 3, namely the cases $M=N / 2$ and $M=N / 4$.

Some numerical experiments disprove a conjecture on the open-type quadrature analogous to Conjecture 2 for closed-type rules. Specifically, the set of 
sample points $\left\{\cos 2 \pi \alpha_{l}\right\}, 1 \leq l \leq 2^{n}-2^{m}-1$, where $1 \leq m \leq n-2$, may yield no positive quadrature rule of open type.

\section{ACKNOWLEDGMENTS}

We are grateful to the referee for valuable comments. We also would like to thank Professor Walter Gautschi for his detailed suggestions for improving the presentation.

\section{BIBLIOGRAPHY}

1. R. Askey, Positivity of the Cotes numbers for some Jacobi abscissae II, J. Inst. Math. Appl. 24 (1979), 95-98.

2. M. Branders and R. Piessens, An extension of Clenshaw-Curtis quadrature, J. Comput. Appl. Math. 1 (1975), 55-65.

3. H. Brass, Eine Fehlerabschätzung für positive Quadraturformeln, Numer. Math. 47 (1985), 395-399.

4. C. W. Clenshaw and A. R. Curtis, A method for numerical integration on an automatic computer, Numer. Math. 2 (1960), 197-205.

5. G. Criscuolo, G. Mastroianni, and D. Occorsio, Convergence of extended Lagrange interpolation, Math. Comp. 55 (1990), 197-212.

6. P. J. Davis and P. Rabinowitz, Methods of numerical integration, 2nd ed., Academic Press, Orlando, 1984.

7. S. Elhay and J. Kautsky, Algorithm 655-IQPACK: FORTRAN subroutines for the weights of interpolatory quadratures, ACM Trans. Math. Software 13 (1989), 399-415.

8. D. Elliott, Truncation errors in two Chebyshev series approximations, Math. Comp. 19 (1965), 234-248.

9. H. Engels, Numerical quadrature and cubature, Academic Press, London, 1980.

10. S. Filippi, Angenäherte Tschebyscheff-Approximation einer Stammfunktion-eine Modifikation des Verfahrens von Clenshaw und Curtis, Numer. Math. 6 (1964), 320-328.

11. W. Gautschi and S. E. Notaris, Gauss-Kronrod quadrature formulae for weight functions of Bernstein-Szegö type, J. Comput. Appl. Math. 25 (1989), 199-224.

12. W. Gautschi and T. Rivlin, A family of Gauss-Kronrod quadrature formulae, Math. Comp. 51 (1988), 749-754.

13. W. M. Gentleman, Implementing Clenshaw-Curtis quadrature II. Computing the cosine transformation, Comm. ACM 15 (1972), 343-346.

14. G. Hämmerlin and K. H. Hoffmann, Numerische Mathematik, Springer-Verlag, Berlin, Heidelberg, 1989.

15. T. Hasegawa, T. Torii, and I. Ninomiya, Generalized Chebyshev interpolation and its application to automatic quadrature, Math. Comp. 41 (1983), 537-553.

16. T. Hasegawa, T. Torii, and H. Sugiura, An algorithm based on the FFT for a generalized Chebyshev interpolation, Math. Comp. 54 (1990), 195-210.

17. P. Henrici, Applied and computational complex analysis, Vol.2, Wiley, New York, 1977.

18. J. P. Imhof, On the method for numerical integration of Clenshaw and Curtis, Numer. Math. 5 (1963), 138-141.

19. J. N. Lyness, The calculation of Fourier coefficients by the Möbius inversion of the Poisson summation formula. Part I. Functions whose early derivatives are continuous, Math. Comp. 24 (1970), 101-135.

20. G. Monegato, Positivity of the weights of extended Gauss-Legendre quadrature rules, Math. Comp. 32 (1978), 243-245.

21. T. N. L. Patterson, An algorithm for generating interpolatory quadrature rules of the highest degree of precision with preassigned nodes for general weight functions, ACM Trans. Math. Software 15 (1989), 123-136. 
22. F. Peherstorfer, Linear combination of orthogonal polynomials generating positive quadrature formulas, Math. Comp. 55 (1990), 231-241.

23. P. Rabinowitz, J. Kautsky, S. Elhay, and J. C. Butcher, On sequences of imbedded integration rules, Numerical Integration: Recent Developments, Software and Applications (P. Keast and G. Fairweather eds.), Reidel, Dordrecht, 1987, pp. 113-139.

24. T. Torii, Fast Fourier sine and cosine transform based on the midpoint rule, J. Inform. Process. 15 (1974), 670-679, in Japanese.

Department of INFORMation Science, Faculty of Engineering, Fukui University, FuKUI, 910, JAPAN

E-mail address: hasegawa@fuis.fuis.fukui-u.ac.jp

DEPARTMENT OF INFORMATION ENGINEERING, FACULTY OF ENGINEERING, NAgOYA UNIVERSITY, NAGOYA, 464-01, JAPAN

DEPARTMENT OF INFORMATION ENGINEERING, FACULTY OF ENGINEERING, NAGOYA UNIVERSITY, NAGOYA, 464-01, JAPAN 